\title{
Experimental Investigations on the Grouping Behaviour in a Mono- Disperse Droplet Stream
}

\author{
Visakh Vaikuntanathan*1, Kasra Amini ${ }^{1}$, Alumah Arad $^{2}$, David Katoshevski ${ }^{2}$, Barry \\ Greenberg $^{3}$, Bernhard Weigand ${ }^{1}$ \\ ${ }^{1}$ Institute of Aerospace Thermodynamics (ITLR), University of Stuttgart, Stuttgart, Germany \\ ${ }^{2}$ Dept. of Civil and Environmental Engineering, Ben-Gurion University of the Negev, Beer- \\ Sheva, Israel \\ ${ }^{3}$ Aerospace Engineering, Technion - Israel Institute of Technology, Haifa, Israel \\ ${ }^{*}$ Corresponding author email: visakh.vaikuntanathan@itlr.uni-stuttgart.de
}

\begin{abstract}
Clustering of droplets in a spray, referred to as 'droplet grouping', is an important phenomenon which affects droplet vaporization and combustion processes. The factors which govern droplet grouping mechanics are not yet fully understood. In this experimental study we consider a single stream of monodisperse droplets to investigate droplet grouping. A circular cylindrical jet of isopropanol is injected into ambient air through a circular hole under a controlled upstream pressure. The jet is broken into droplets by excitation using a piezo element. A double frequency excitation generates a stream of droplet groups each consisting of two droplets. The droplets are injected into a quiescent ambient air at velocities much greater than their terminal velocity of free fall. From high-resolution images of the droplet stream, the evolution of the intra- and inter-group distances is measured. It is observed that, for the experimental conditions explored here, the droplets within a group approach each other, collide, coalesce, and exhibit droplet shape oscillations. The characteristics of the droplet grouping process are reported.
\end{abstract}

\section{Keywords}

Droplet dynamics; droplet grouping; droplet collision; droplet coalescence.

\section{Introduction}

A liquid spray, defined as a collection of liquid droplets moving in an ambient medium, is seen in many scenarios ranging from natural ones (for example, rain) to artificial ones in technical applications (for example, fuel sprays in engines, agricultural sprays, medicinal sprays, and coolant sprays). In such sprays, there is a tendency of droplets which were initially farther apart to come closer together and form droplet clusters. This phenomenon is referred to as 'droplet grouping' or 'droplet clustering' $[1,2]$. Droplet grouping could have both detrimental and beneficial effects, depending on the scenario under consideration. For example, grouping of droplets in fuel sprays could affect the vaporization of individual droplets in the group [3]. On the other hand, grouping and coalescence of cough or sneeze droplets could lead to a faster settling down of potentially virus-laden cough/sneeze droplets and prevent their transmission through advection in air [4]. The conditions at which droplet grouping is observed and the mechanisms responsible for the formation of such droplet groups are not yet clearly understood. This sets the broad motivation for this study.

There are very few experimental studies reported in the literature which investigated droplet grouping or clustering in sprays [2,5]. Given the polydisperse nature and three-dimensionality of such sprays, it is difficult to understand fundamental physical mechanisms responsible for droplet grouping from such studies. Hence, simplified systems have been looked at in the past. One example of such a simplified system is a pair of droplets or solid spheres moving 
vertically under the influence of gravity in an ambient medium. Several such studies focused on such pairs of solid spheres falling from rest inside a viscous medium [6-8]. The Reynolds number, $\operatorname{Re}_{s}=\rho_{\infty} \mathrm{V}_{s, t} \mathrm{D}_{s} / \mu_{\infty}$, calculated based on the sphere size (diameter, $\mathrm{D}_{s}$ ), ambient fluid properties (density, $\rho_{\infty}$; dynamic viscosity, $\left.\mu_{\infty}\right)$, and terminal velocity of sphere $\left(\mathrm{V}_{\mathrm{s}, \mathrm{t}}\right)$, is small (of the order of 1 or less) in such studies, justifying the use of Stokes flow approximation. These studies revealed that the drag force acting on the trailing sphere is reduced compared to that acting on the leading sphere [6-8]. This asymmetry in the force acting on the leading and trailing spheres then leads to the grouping of the spheres over time. In sprays, however, the droplets are injected at velocities much greater than their terminal velocity of free fall. For a typical agricultural aqueous spray, the droplet size (diameter, $\left.D_{d}\right)$ and velocity $\left(V_{d}\right)$ are of the order of $100 \mu \mathrm{m}$ and $10 \mathrm{~m} / \mathrm{s}$ [9] respectively, whereas the terminal velocity $\left(\mathrm{V}_{\mathrm{d}, \mathrm{t}}\right)$ of an aqueous/water droplet of similar size in ambient air in Stokes flow regime is of the order of 0.1 $\mathrm{m} / \mathrm{s}$. Hence, the Reynolds number of the spray droplets $\left(R e=\rho_{\infty} V_{d} D_{d} / \mu_{\infty}\right)$ is two orders of magnitude larger than that in typical Stokes flow regime. Under such conditions it is still unclear if the observations from Stokes flow regime are still valid. Hence it is important to investigate the phenomenon of droplet grouping at these higher Reynolds numbers (far away from Stokes flow regime). This is the focus of the study reported here.

A simplified model system consisting of a unidirectional stream of monodisperse droplets is considered in this study. The droplet stream is generated through a controlled excitation of a liquid jet issuing from a droplet generator. This excitation process generates droplets which are slightly closer to their neighbouring droplet in one direction (say, upstream) compared to that in the other direction (say, downstream). The evolution of the droplets in the stream is followed in space-time to understand the mechanics of grouping. A previous experimental study from our research group reported experiments at a Reynolds number of around 112 [1]. Here we explore a higher Reynolds number of around 492. Section II details the experimental set-up and methodology employed in this investigation. Section III presents and discusses the main results on the mechanics of grouping. A summary of the main findings and an outlook for future work are provided in Sec. IV.

\section{Experimental details}

The experimental set-up consists of a droplet generator, flow lines for the droplet liquid and compressed air, a double frequency generator, and an imaging system. For details of the experimental set-up, please refer to Fig. 1 in [1]. The relevant details and some modifications of the set-up reported in [1] will be outlined here.

A. Liquid and flow line: The droplet liquid in this study is isopropanol (2-propanol). The physical properties of 2-propanol at room temperature are as follows: density, $\rho_{d}=786 \mathrm{~kg} / \mathrm{m}^{3}$; dynamic viscosity, $\mu_{d}=2.38 \mathrm{mPa}-\mathrm{s}$; and surface tension, $\sigma_{d}=21.7 \mathrm{mN} / \mathrm{m}$. The properties of the ambient air at room temperature are as follows: density, $\rho_{\infty}=1.2 \mathrm{~kg} / \mathrm{m}^{3}$; dynamic viscosity, $\mu_{\infty}=0.018 \mathrm{mPa}$-s. The liquid is stored in an air-pressurized stainless-steel tank. The pressurized liquid is taken out of the tank through on/off valves, filter to arrest dirt/particles in the flow, control valve to adjust the mass flow rate, pressure gauges to monitor the flow pressure, and into the droplet generator body through flexible hoses.

B. Droplet generator: The body of the droplet generator consists of a cylindrical metallic chamber which could be fitted with a removable unit in which an orifice of appropriate size could be mounted (see Figs. 1 and 2 in [1]). In this study, an orifice of diameter, $D_{i}=118 \pm 3$ 
$\mu \mathrm{m}$ is used. Figure 1(a) shows an optical microscopic image of the orifice. The droplet generator with this orifice is calibrated for its mass flow rate. This is carried out by first setting the upstream flow pressure, $\Delta \mathrm{P}_{\mathrm{i}}$ at desired values (ranging from 0.4 to 4 bar) by regulating the liquid storage tank pressure. Then the liquid flow pressure is monitored with the pressure gauge installed in the liquid flow line upstream of the droplet generator. Afterwards, for each value of $\Delta \mathrm{P}_{\mathrm{i}}$, the liquid mass $\Delta \mathrm{m}$ issuing out of the droplet generator over a fixed time interval, $\Delta \mathrm{t}$ of $9-11 \mathrm{~s}$ is measured by collecting the liquid and measuring the collected liquid mass using a weighing balance. The average mass flow rate, $m$ corresponding to the set value of $\Delta \mathrm{P}_{\mathrm{i}}$ is then calculated as $\Delta \mathrm{m} / \Delta \mathrm{t}$. Figure 1 (b) shows the flow calibration curve (m versus $\Delta \mathrm{P}_{i}{ }^{0.5}$ ) of the droplet generator with this orifice. The flow calibration curve helps to repeatedly set a desired mass flow rate condition. The body of the droplet generator also houses a piezo element to impart controlled external perturbations to the fluid flow with the help of a double frequency generator (for details about the operation of the double frequency generator, please refer to [1]).

(a)

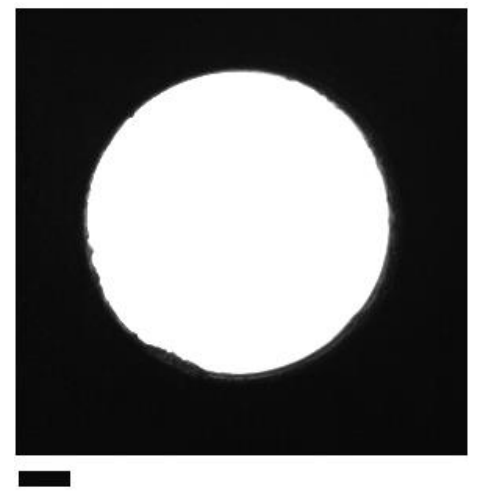

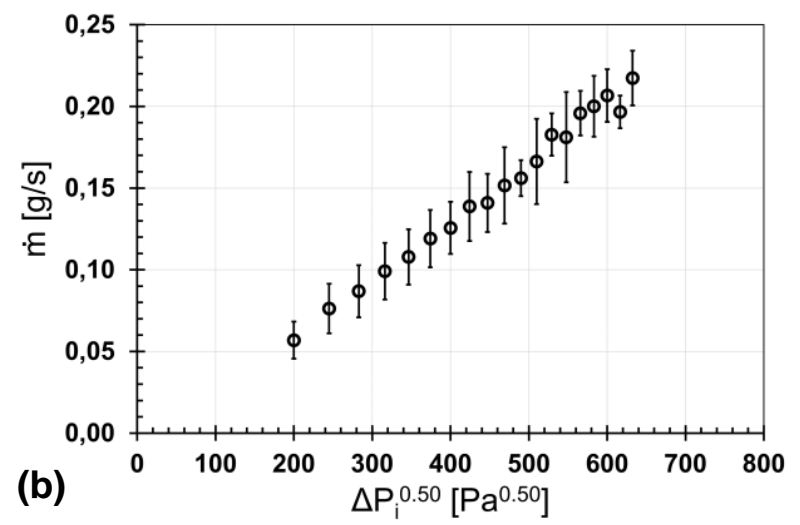

Figure 1. (a) Optical microscopic image of orifice with diameter, $D_{i}=118 \pm 3 \mu \mathrm{m}$. Scale bar: $20 \mu \mathrm{m}$. (b) Flow calibration curve of droplet generator with the orifice shown in (a).

C. Imaging system: The imaging system consists of a camera (Model UI-3180CP-C-HQ R2 from IDS), an objective (Schneider Kreuznach 4/150 APO-Componon HM), and a light source (Nanolite). The light source is controlled by the low frequency output from the double frequency generator such that the motion of the droplets in the stream can be 'frozen' in time through the nanosecond pulses of the light source. The images of the droplet stream, such as in Fig. 2, are acquired with the imaging system in a backlit shadowgraphy mode in the form of a video. The software uEye Cockpit (from IDS) is used for capturing and saving the acquired video/movie frames. The pixel size in the conjugated object plane of the imaging system is approximately $2 \mu \mathrm{m}$.

D. Experimental methodology and post-processing: Before starting the experiments, the orifice is cleaned with the help of pressurized air to remove any dust particles. It is also ensured visually that the flow line is devoid of any air bubbles and, if present, are not communicated to the droplet generator. The liquid is injected out of the orifice in the droplet generator in the form of a liquid jet into the quiescent ambient. Once the external frequency, $f_{s}$ at which such a mono-disperse droplet stream forms is identified, the double frequency generator is operated with the lower frequency $f_{1}=f_{s} / n$ where $n$ is the number of droplets desired in a group (here, $n=2$ ), and the higher frequency $f_{2}>f_{1}$. In the present study, $f_{1}=26 \mathrm{kHz}$ and $f_{2}=56 \mathrm{kHz}$. Note that the frequency $f_{2}$ is set slightly greater than $52 \mathrm{kHz}$, so that the distances $a_{1}$ and $a_{2}$ between 
droplets in the stream (see Fig. 2) are slightly different. Meeting this condition, in the current experiments, a stable droplet stream was observed for $f_{2}=56 \mathrm{kHz}$. At a given axial location, $\ell$ from the droplet generator exit, multiple images of the droplet stream are captured in the form of a video. Typically, around 100 images are captured and saved at each axial location. The droplet generator, mounted on a vertical traverse system, could be moved up and down to vary the observed axial location. A similar procedure is repeated for other axial locations till the entire process including the liquid jet injection at the droplet generator exit, liquid jet breakup and droplet stream formation, droplet grouping process, droplet collision, droplet coalescence, and droplet shape oscillations are captured.

A customized MATLAB code is used to convert the shadowgraph image captured by the experiment to a grey-scale 2D array, which is subjected to black/white threshold and converted to a binary image. A planar scanning of the binary array identifies the droplets' peripheries to extract the droplets' peripheral points. From this droplet shape data, the physically relevant parameters such as the droplet size, the axial location of the droplet centre and the interdroplet spacing are calculated.

\section{Results and Discussion}

\section{A. Visual observations}

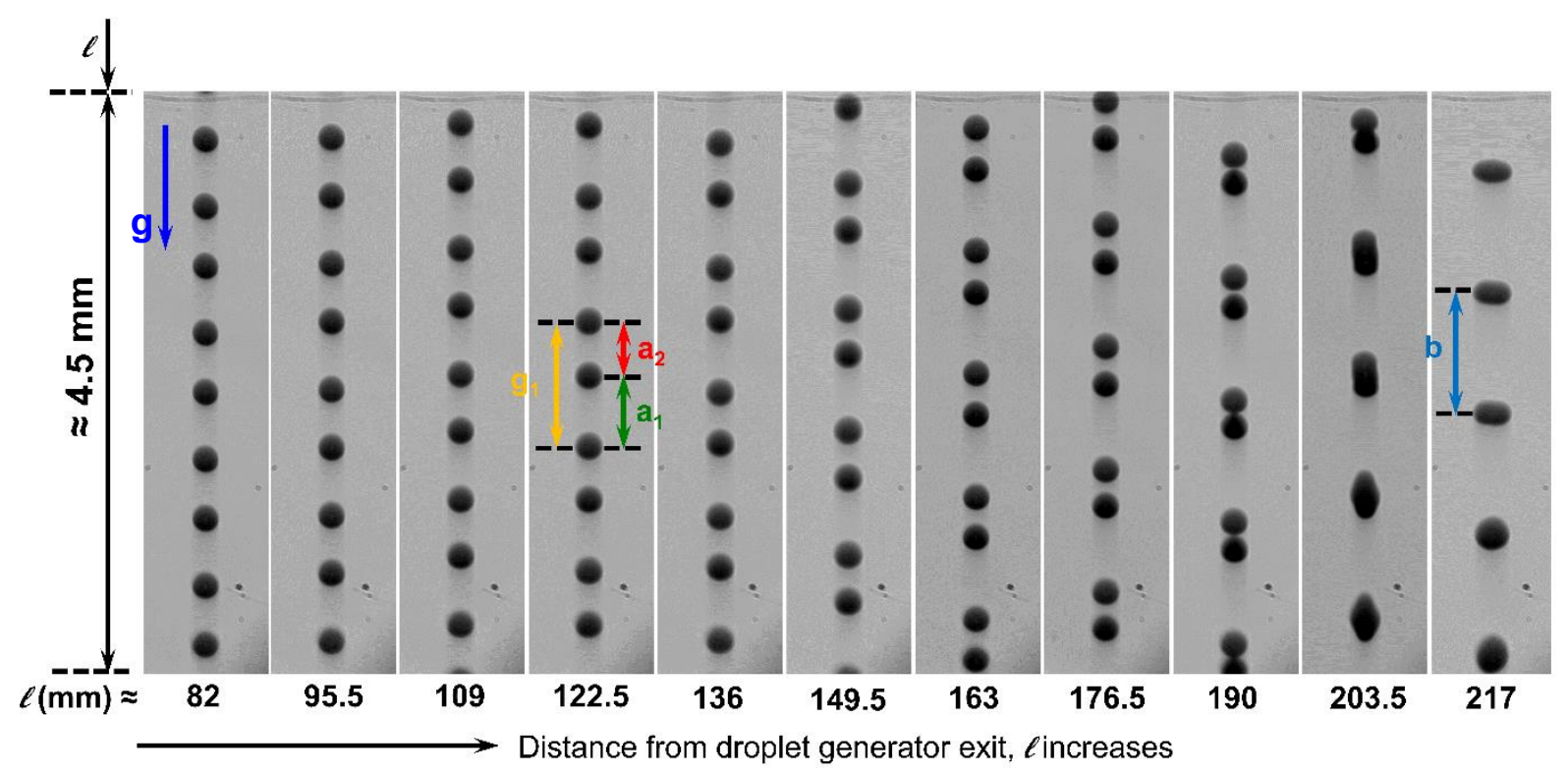

Figure 2. Image sequence showing the droplet stream at different axial locations. The flow direction of the droplet stream as well as the direction of gravity is from top to bottom, as shown by the vertical blue arrow in the first image strip. The axial location, $\ell$ of the top of an image strip is mentioned at the bottom of that image strip. From left to right in the image sequence, $\ell$ increases. Flow conditions: average droplet diameter, $D_{d}=236 \pm 6 \mu \mathrm{m}$; initial droplet velocity, $\mathrm{V}_{\mathrm{d} 0}=31.6 \pm 0.1 \mathrm{~m} / \mathrm{s}$. The relevant inter-droplet distances (during grouping process: $\mathrm{a}_{1}$ and $\mathrm{a}_{2}$; after droplet coalescence: b), and inter-group distance (group period, $g_{1}$ ) are highlighted.

Figure 2 shows a typical image sequence of a single stream of isopropanol droplets from the droplet generator with the orifice shown in Fig. 1(a). The flow pressure measured upstream of the droplet generator is $3.4 \mathrm{bar}\left(\Delta \mathrm{P}_{\mathrm{i}}{ }^{0.50}=583 \mathrm{~Pa}^{0.50}\right.$ in Fig. $\left.1(\mathrm{~b})\right)$. This corresponds to a mass flow rate of around $0.20 \mathrm{~g} / \mathrm{s}$ (from Fig. 1(b)). From the left to the right in Fig. 2, the axial location $\ell$ measured from the exit of the droplet generator $(\ell=0 \mathrm{~mm})$ increases from around $82 \mathrm{~mm}$ to around $217 \mathrm{~mm}$. The values of $\ell$ noted at the bottom of each image strip in Fig. 2 correspond 
to the location of the top of that image strip from the droplet generator exit. The flow conditions, defined at an axial location where monodisperse droplets are formed in the droplet stream, will be referred to as the initial conditions. For the case presented in Fig. 2, these can be summarized as follows: droplet diameter, $D_{d}=236 \pm 6 \mu \mathrm{m}$; droplet velocity, $V_{d 0}=31.6 \pm 0.1 \mathrm{~m} / \mathrm{s}$. Please note that the droplet diameter mentioned here is the average of droplet diameters at all axial locations; we do not observe a significant decrease in droplet diameter due to evaporation during the time scale of the droplet stream flow considered here $(<\approx 7 \mathrm{~ms})$. The corresponding Reynolds number, $R e=\rho_{\infty} \mathrm{V}_{\mathrm{d} 0} \mathrm{D}_{\mathrm{d}} / \mu_{\infty} \approx 492 \pm 13$. This is a higher Re compared to that in the previous study from our research group, where Re was $\approx 112$ [1]. As $\ell$ increases, a pair of droplets in the droplet stream gradually approach each other creating groups of two droplets in the stream. Further downstream, with increase in $\ell$, the droplets in each group collide with each other, leading to their coalescence to form larger droplets. Due to the relative velocity with which the droplets in a group collide, the coalescence process leads to shape oscillations of the larger coalesced droplet. When the distance between the droplets is small, as in the image strips with $\ell \approx 190 \mathrm{~mm}$ and $\ell \approx 203.5 \mathrm{~mm}$, the line connecting the droplet centres rotate away from the line of motion of the droplet stream. This could be because of an asymmetry in the wake of the leading droplet in a group in the vicinity of the droplet surface. This could lead to an asymmetric draining of the air film between the two droplets when they approach close to each other. This asymmetry in turn is reflected as the tilting of the line of droplet centres with respect to the vertical direction of motion of the droplet stream. The images shown in Fig. 2 clearly show that droplet grouping is observed even at this high Reynolds number.

The motion of droplets in this droplet stream could be classified into two broad stages: (1) grouping stage in which groups of two droplets are clearly seen in the droplet stream (up to the image strip with $\ell \approx 190 \mathrm{~mm}$ in Fig. 2), and (2) coalescence stage in which the droplets in a group collide with each other, coalesce to form larger droplets, and exhibit shape oscillations (beyond the image strip with $\ell \approx 190 \mathrm{~mm}$ in Fig. 2). In the next sub-section, the motion of droplets in the grouping stage is quantitatively discussed in terms of the temporal evolution of inter-group and inter-droplet distances marked as $g_{1}, a_{1}$ and $a_{2}$ in Fig. 2.

\section{B. Grouping mechanics}

Figure 3(a) shows the axial variation of the inter-droplet distances $a_{1}$ and $a_{2}$. Please note that at the axial location of the centre of a droplet in the stream, the corresponding inter-droplet distances are measured downstream from that axial location. Also, the data in Fig. 3(a) do not start at $\ell=0$ since droplets are formed from liquid jet at an axial location $\ell>0$. It is evident that $a_{1}$ increases with $\ell$ whereas $a_{2}$ decreases with $\ell$. Both the curves exhibit an exponential behaviour with the axial location $\ell$. The curves are stopped when the droplets in a group contact each other $\left(\mathrm{a}_{2}\right.$ approaching $\left.\mathrm{D}_{\mathrm{d}}\right)$.

To convert the axial location $\ell$ to an equivalent time t, we follow a methodology as reported in the previous study from our research group [1]. For this, the velocity of droplet in the stream at an axial location $\ell$ is expressed as $V_{d}=d \ell / d t$. The velocity of the droplet at a particular axial location can be expressed as the product of the lower frequency of excitation, $f_{1}$ and the spatial period of the droplet group at this axial location, $g_{1}=a_{1}+a_{2}: V_{d}=f_{1} g_{1}$. This gives $f_{1} g_{1}=d l / d t$. Rearranging the terms gives the following equation connecting infinitesimal increments in axial location $\mathrm{d} \ell$ and those in time $\mathrm{dt}$ : $\mathrm{dt}=\left(\mathrm{f}_{1} \mathrm{~g}_{1}\right)^{-1} \mathrm{~d} \boldsymbol{l}$. The velocity of the droplet $\left(\mathrm{f}_{1} \mathrm{~g}_{1}\right)$ and, hence, $\left(f_{1} g_{1}\right)^{-1}$ are functions of $\ell$. Plotting the measurements of $\left(f_{1} g_{1}\right)^{-1}$ versus $\ell$ and fitting this trend with a linear function of $\ell$ of the form, $\left(f_{1} g_{1}\right)^{-1}=e_{1}+e_{2} \ell$ would give 
$d t=\left(e_{1}+e_{2} \ell\right) d \ell$.

Equation (1) can be directly integrated with the condition that at $\ell=0, \mathrm{t}=0$ to give the following equation to convert $\ell$ to $t$.

$\mathrm{t}=\mathrm{e}_{1} \ell+\left(\mathrm{e}_{2} / 2\right) \boldsymbol{\ell}^{2}$.

Figure 3(b) shows the variation of $\left(f_{1} g_{1}\right)^{-1}$ with $\ell$ for the data presented in Fig. 3(a). There is a linearly increasing trend of $\left(f_{1} g_{1}\right)^{-1}$ with $\ell$. This indicates that the droplet velocity $\left(f_{1} g_{1}\right)$ has a decreasing trend with $\boldsymbol{\ell}$. In other words, the droplets decelerate as they flow downstream. This observation is consistent with the previously reported finding for the case of a lower $\operatorname{Re}[1]$. A linear best-fit of the experimental data in Fig. $3(\mathrm{~b})$ gives the values of the coefficients: $e_{1}=$ $0.0307, \mathrm{e}_{2}=0.0104$. The relative root mean square deviation between the data and the fitted line is less than $0.6 \%$. This further justifies the use of a linear fit to the data in Fig. 3(b). Using the calculated values of the coefficients $e_{1}$ and $e_{2}$ in Eq. (2) enables a conversion of the plots from axial trends to temporal trends.
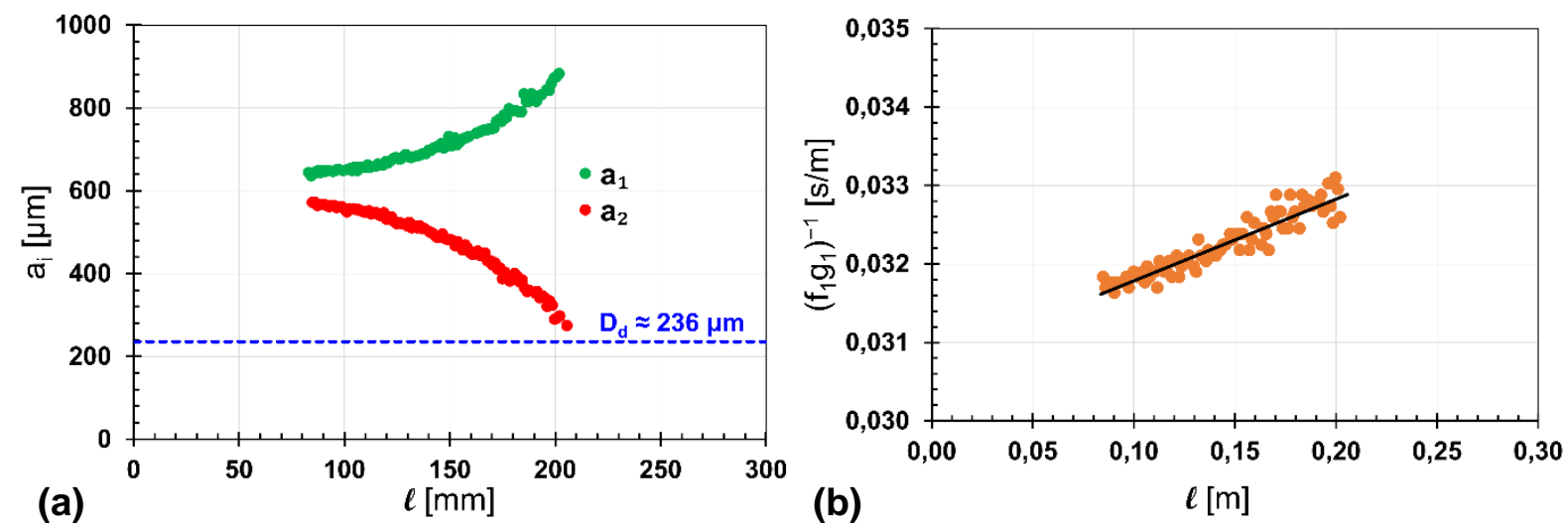

Figure 3. (a) Plot showing the axial variation of the inter-droplet distances, $a_{1}$ and $a_{2}$, corresponding to the experimental condition presented in Fig. 2. (b) Plot showing the axial variation of the parameter $\left(f_{1} g_{1}\right)^{-1}$ corresponding to the experimental data presented in (a). Please note that here $f_{1} g_{1}$ is the droplet velocity. The line shown in the plot is the best fit straight line, of the form $\left(f_{1} g_{1}\right)^{-1}=e_{1}+e_{2} \ell$, to the experimental data. $e_{1}=0.0307, e_{2}$ $=0.0104$.

Figure 4(a) shows the temporal variation of the inter-droplet distances $a_{1}$ and $a_{2}$ corresponding to the data in Fig. 3(a). The entire grouping phenomenon, starting from the formation of groups of two droplets in the monodisperse droplet stream to the contact between the droplets in a group, occurs over a time duration of approximately $4.06 \mathrm{~ms}$. The time at which the droplets in a group come in contact with each other, $t_{0} \approx 6.65 \mathrm{~ms}$. The curves plotted in Fig. 4(a) correspond to the exponential best fit to the experimental data of $a_{1}$ and $a_{2}$. The equations of the best fit exponential curves are as follows.

$a_{1}=7.710 \exp (552.83 t)+608$,

$\mathrm{a}_{2}=-8.615 \exp (566.04 \mathrm{t})+608$,

where $a_{1}$ and $a_{2}$ are expressed in $\mu \mathrm{m}$ and $t$ is in seconds. The above equations are valid for the experimental data at $t \in[2.59,6.65] \mathrm{ms}$. The coefficients of determination, $\mathrm{R}^{2}$ of these best fit curves are 0.9899 and 0.9935 , respectively.

The relative velocity with which the leading droplet in a group approaches the trailing droplet in the neighbouring downstream group, $\Delta \mathrm{V}_{\mathrm{d}, 1}=\mathrm{V}_{\mathrm{d}, \mathrm{t}}{ }^{\prime}-\mathrm{V}_{\mathrm{d}, \mathrm{l}}$ can be calculated as the time derivative of $a_{1}$ from Eq. (3a). This is given in Eq. (4a). $V_{d, l}$ and $V_{d, t}$ are the velocities of the 
leading droplet in a group and the trailing droplet in the neighbouring downstream group, respectively.

$\Delta \mathrm{V}_{\mathrm{d}, 1} \equiv \mathrm{V}_{\mathrm{d}, \mathrm{t}}{ }^{\prime}-\mathrm{V}_{\mathrm{d}, \mathrm{l}}=\mathrm{da}_{1} / \mathrm{dt}=4.262 \exp (552.83 \mathrm{t})$,

where $\Delta \mathrm{V}_{\mathrm{d}, 1}$ is expressed in $\mathrm{mm} / \mathrm{s}$ and $\mathrm{t}$ is expressed in seconds.

Similarly, the relative velocity with which the droplets in a group approach each other $\Delta \mathrm{V}_{\mathrm{d}, 2}=$ $\mathrm{V}_{\mathrm{d}, \mathrm{I}}-\mathrm{V}_{\mathrm{d}, \mathrm{t}}$ can then be calculated as the time derivative of $\mathrm{a}_{2}$ from Eq. (3b). This is given in Eq. (4b). $V_{d, t}$ is the velocity of the trailing droplet in a group.

$\Delta \mathrm{V}_{\mathrm{d}, 2} \equiv \mathrm{V}_{\mathrm{d}, \mathrm{I}}-\mathrm{V}_{\mathrm{d}, \mathrm{t}}=\mathrm{da}_{2} / \mathrm{dt}=-4.876 \exp (566.04 \mathrm{t})$,

where $\Delta \mathrm{V}_{\mathrm{d}, 2}$ is expressed in $\mathrm{mm} / \mathrm{s}$ and $\mathrm{t}$ is expressed in seconds.

Equations (4a) and (4b) can be used to calculate the temporal variation of the velocities of trailing and leading droplets in the droplet groups, with the 'initial condition' of $\mathrm{V}_{\mathrm{d}, \mathrm{t}}(\mathrm{at} t=2.59$ $\mathrm{ms}) \approx \mathrm{V}_{\mathrm{d} 0} \approx 31.6 \mathrm{~m} / \mathrm{s}$.
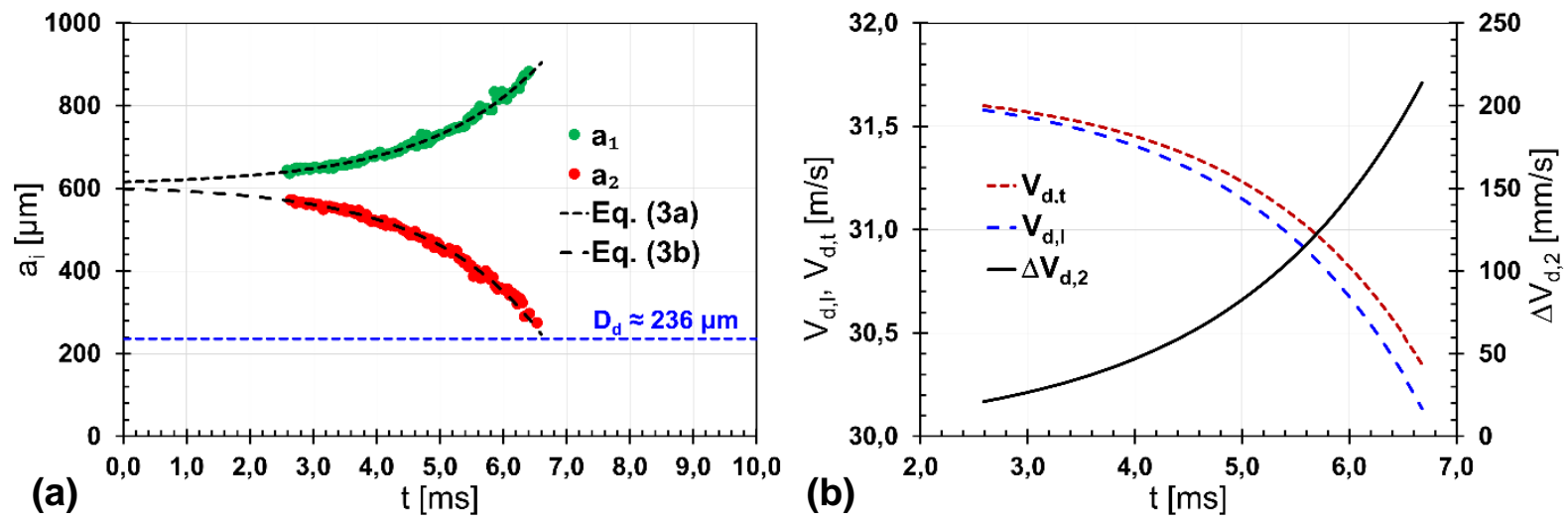

Figure 4. (a) Plot showing the temporal variation of inter-droplet distances corresponding to the data in Fig. 3(a). Please refer to the text for the details on conversion of the data from Fig. 3(a). The curves shown are the best fit exponential curves to the experimental data, given by Eq. (3a) and Eq. (3b). (b) Plot showing the temporal variation of the velocities of the trailing and leading droplets $\left(\mathrm{V}_{\mathrm{d}, \mathrm{t}}\right.$ and $\mathrm{V}_{\mathrm{d}, \mathrm{I}}$, respectively) in the droplet groups corresponding to the experimental data presented in (a). The relative velocity between the droplets in a group, $\Delta \mathrm{V}_{\mathrm{d}, 2}(\mathrm{Eq}$. (4b)) is also plotted. Equations (4a) and (4b) are used to obtain the plots. Please refer to the text for details of the calculation.

Figure $4(\mathrm{~b})$ shows the trends of $\mathrm{V}_{\mathrm{d}, \mathrm{t}}$ and $\mathrm{V}_{\mathrm{d}, \mathrm{I}}$ with time based on these calculations. It is evident that the leading droplet decelerates faster than the trailing droplet. This shows that the net decelerating force (here, mainly the drag force) acting on the leading droplet is more than that on the trailing droplet. The resulting difference in velocities of the droplets in a group causes the droplets to approach each other. This is consistent with the experimental findings at a lower Re reported previously [1] as well as with theoretical studies with a pair of spheres/droplets in Stokes flow regime [6-8]. The difference in the deceleration of the droplets in a group is possibly due to the interaction of the trailing droplet with the wake of the leading droplet.

The relative velocity between the leading and trailing droplets in a group, $\Delta \mathrm{V}_{\mathrm{d}, 2}$ increases with time (see Fig. 4(b)). The relative velocity between the droplets in a group at the time instant when the droplets in a group contact each other, $t_{0} \approx 6.65 \mathrm{~ms}$ can be calculated from Eq. (4b) as $\left(\Delta \mathrm{V}_{\mathrm{d}, 2}\right)_{\mathrm{o}} \approx-0.210 \mathrm{~m} / \mathrm{s}$. This gives a collision Weber number, We $=\rho_{\mathrm{d}}\left(\Delta \mathrm{V}_{\mathrm{d}, 2}\right)_{\mathrm{o}}{ }^{2} \mathrm{D}_{\mathrm{d}} / \sigma_{\mathrm{d}} \approx 0.377$. The critical collision Weber number below which two droplets undergoing head-on collision will coalesce to form a larger droplet is given by [10]: $\mathrm{We}_{c}=30 \mathrm{Oh}_{d}+15$. Here $\mathrm{Oh}_{d}=$ 
$16 \sqrt{ } 2 \mu_{d} / \sqrt{ }\left(\rho_{d} \sigma_{d} D_{d}\right)$ is the droplet Ohnesorge number. For the current experimental conditions, $\mathrm{Oh}_{\mathrm{d}} \approx 0.85, \mathrm{We}_{\mathrm{c}} \approx 40.5$. This means that $\mathrm{We}<\mathrm{We}_{\mathrm{c}}$ and, hence, the colliding droplets are predicted to undergo coalescence. This is consistent with the experimental observations in Fig. 2.

\section{Conclusions}

An experimental investigation of the phenomenon of droplet grouping in a single monodisperse stream of isopropanol droplets was presented. The flow calibration curve of the droplet generator with the desired injector hole enables a proper control and easy setting of the desired mass flow rate out of the droplet generator. The droplet Reynolds number was around 492. Droplet grouping was also observed in this case of a relatively high Re. This strengthens the experimental observations in a previous study [1] at a lower Re of around 112. The droplet grouping mechanics in the single droplet stream was studied through the axial and temporal variations of inter-droplet and inter-group distances. The inter-droplet distances exhibited exponential trends in time, consistent with the finding at a lower Re. The velocities of the trailing and leading droplets decreased with time highlighting the importance of deceleration due to drag force. The leading droplet decelerated faster than the trailing droplet, pointing at an asymmetry in the drag force experienced by the droplets in a group. Using the relative velocity with which the droplets in a group approached each other in a critical Weber number criterion for binary droplet collisions, it was possible to predict the coalescence of droplets upon collision. It is confirmed that the phenomenon of grouping, classically reported for Stokes flow $(\operatorname{Re}<1)$ around solid spheres [6-8], seems to be occurring also for $\operatorname{Re}>1$ in the current study with $\mathrm{Re} \approx 492$ and the previous study with $\mathrm{Re} \approx 112$. The question which remains is: how grouping phenomenon, theoretically predicted at low $R e$, is also seen at higher Re? There are hints from this and the previous study which point at the asymmetry in drag force on the droplets as a potential answer. There is an ongoing effort which tries to clarify this with additional experiments, analytical models, and numerical simulations.

\section{Acknowledgments}

The authors gratefully acknowledge the financial support from the Deutsche Forschungsgemeinschaft (DFG) through the project 'Investigation of droplet motion and grouping' (Project number 409029509, WE2549/41-1).

\section{References}

[1] Roth, N., Gomaa, H., Livne, A., Katoshevski, D., Weigand, B., Sep. 6.-8. 2017, $28^{\text {th }}$ European Conference on Liquid Atomization and Spray Systems.

[2] Heinlein, J., and Fritsching, U., 2006, Exp. Fluids, 40, pp. 464-472.

[3] Katoshevski, D., Shakked, T., Sazhin, S. S., Crua, C., Heikal, M. R., 2008, Int. J. Heat Fluid Flow, 29, pp. 415-426.

[4] Bourouiba, L., 2020, JAMA, 323 (18), pp. 1837-1838.

[5] Manish, M., and Sahu, S., 2018, Phys. Fluids, 30, pp. 123305.

[6] Stimson, M., and Jeffery, G. B., 1926, Proc. R. Soc. A., 111, pp. 110-116.

[7] Happel, J., and Pfeffer, R., 1960, AIChE J., 6, pp. 129-133.

[8] Steinberger, E. H., Pruppacher, H. R., Neiburger, M., 1968, J. Fluid Mech., 34, pp. 809819.

[9] Nuyttens, D., De Schampheleire, M., Verboven, P., Brusselman, E., Dekeyser, D., 2009, Trans. ASABE, 52(5), pp. 1471-1480.

[10] Qian, J., and Law, C. K., 1997, J. Fluid Mech., 331, pp. 59-80. 